

DO RIO DE JANEIRO

Rodrigo da Silva Ferreira

\title{
Uma Abordagem Multiescalar, Multicritério para a Segmentação de Imagens
}

Dissertação de Mestrado

Dissertação apresentada como requisito parcial para obtenção do grau de Mestre pelo Programa de PósGraduação em Engenharia Elétrica da PUC-Rio.

Orientador: Prof. Raul Queiroz Feitosa Co-Orientador: Prof. Gilson A. O. P. Costa

Rio de Janeiro

Abril de 2011 


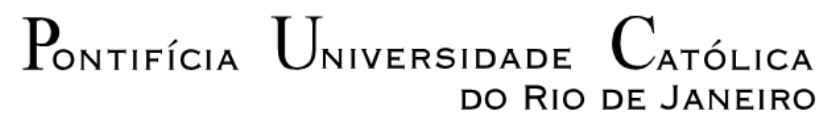

DO RIO DE JANEIRO

Rodrigo da Silva Ferreira

\title{
Uma Abordagem Multiescalar, Multicritério para a \\ Segmentação de Imagens
}

Dissertação apresentada como requisito parcial para obtenção do grau de Mestre pelo Programa de PósGraduação em Engenharia Elétrica da PUC-Rio. Aprovada pela Comissão Examinadora abaixo assinada.

\author{
Prof. Raul Queiroz Feitosa \\ Orientador \\ Departamento de Engenharia Elétrica - PUC-Rio
}

Prof. Gilson Alexandre Ostwald Pedro da Costa

Co-Orientador

Departamento de Engenharia Elétrica - PUC-Rio

Profa. Leila Maria Garcia Fonseca

INPE

Prof. Sidnei Paciornik

Departamento de Engenharia de Materiais - PUC-Rio

Prof. José Eugenio Leal

Coordenador Setorial do Centro

Técnico Científico - PUC-Rio

Rio de Janeiro, 15 de abril de 2011 
Todos os direitos reservados. É proibida a reprodução total ou parcial do trabalho sem autorização da universidade, do autor e do orientador.

\section{Rodrigo da Silva Ferreira}

É formado em Engenharia Elétrica com ênfase em Sistemas e Computação pela Universidade do Estado do Rio de Janeiro e atualmente é aluno de mestrado no programa de Engenharia Elétrica da PUC-Rio. Desenvolve pesquisas na área de Visão Computacional, principalmente sobre interpretação automática de imagens baseada em conhecimento.

Ficha Catalográfica

Ferreira, Rodrigo da Silva

Uma abordagem multiescalar, multicritério para a segmentação de imagens / Rodrigo da Silva Ferreira ; orientador: Raul Queiroz Feitosa ; co- orientador: Gilson A. O. P. Costa. -2011.

113 f. : il. (color.) ; 29,7 cm

Dissertação (mestrado)-Pontifícia Universidade Católica do Rio de Janeiro, Departamento de Engenharia Elétrica, 2011.

Inclui bibliografia

1. Engenharia elétrica - Teses. 2. Processamento de imagens. 3. Segmentação de imagens. 4. Avaliação da qualidade da segmentação. 5. Atributos de forma. I. Feitosa, Raul Queiroz. II. Costa, Gilson A. O. P. III. Pontifícia Universidade Católica do Rio de Janeiro. Departamento de Engenharia Elétrica. IV. Título.

CDD: 621.3 
A Deus, em primeiro lugar. À minha esposa Mydiã, e à família que juntos estamos construindo. Aos meus pais, família e amigos. 


\section{Agradecimentos}

Agradeço a Deus por mais essa vitória. A cada dia que passa entendo mais o seu amor e perdão.

À minha esposa Mydiã, por esse ainda curto tempo em que tenho tido o privilégio de tê-la ao meu lado.

A toda minha família. Em especial aos meus pais, Antônio e Silvana e aos meus irmãos Rafael e Rômulo.

Aos meus sogros e pastores, Edmundo e Márcia, por tudo o que têm feito, pelo carinho e amor dedicado.

Aos meus orientadores Prof. Raul Queiroz Feitosa e Dr. Gilson A. O. P. Costa pelo apoio e incentivo para a realização deste trabalho.

Ao CNPq e à PUC-Rio, pelos auxílios concedidos sem os quais esse trabalho não poderia ter sido realizado.

A todos os meus amigos e irmãos em Cristo, creio que também somos uma família. 


\section{Resumo}

Ferreira, Rodrigo da Silva; Feitosa, Raul Queiroz; Costa, Gilson A. O. P. Uma Abordagem Multiescalar, Multicritério para a Segmentação de Imagens. Rio de Janeiro, 2011. 113p. Dissertação de Mestrado Departamento de Engenharia Elétrica, Pontifícia Universidade Católica do Rio de Janeiro.

O objetivo geral deste trabalho é avaliar o impacto relativo da utilização de atributos de forma na segmentação de imagens de diferentes características e classes de objeto distintas. Para tanto, uma extensão do método de Segmentação Multiresolução (Baatz00) foi proposta e implementada, permitindo que vários atributos de forma possam ser considerados no processo de crescimento de regiões. Para se selecionar a métrica usada na avaliação da qualidade da segmentação, oito métricas disponíveis na literatura foram consideradas. O desempenho relativo das oito métricas foi verificado experimentalmente e avaliada a correlação entre este desempenho e a percepção humana da qualidade da segmentação. Na sequência, dez atributos de forma foram selecionados. A qualidade das segmentações realizadas considerando um atributo de forma de cada vez foi então comparada com a qualidade de segmentações baseadas somente na cor. Depois disso o impacto da utilização de pares de atributos de forma no processo de segmentação foi avaliado. Os experimentos foram realizados para quinze classes de objetos distintas presentes em doze imagens representativas de áreas de aplicação diferentes - sensoriamento remoto, microscopia e imagens médicas. Os resultados confirmam a importância dos atributos de forma na qualidade da segmentação e suscitam uma discussão sobre trabalhos futuros.

\section{Palavras-chave}

Processamento de Imagens; Segmentação de Imagens; Avaliação da Qualidade da Segmentação; Atributos de Forma. 


\section{Abstract}

Ferreira, Rodrigo da Silva; Feitosa, Raul Queiroz (Advisor); Costa, Gilson A. O. P. (Co-Advisor). A Multiscalar, Multicriteria Approach for Image Segmentation. Rio de Janeiro, 2011. 113p. MSc. Dissertation Departamento de Engenharia Elétrica, Pontifícia Universidade Católica do Rio de Janeiro.

This work's general goal is to evaluate the relative impact of using different morphological attributes on the segmentation of different images and object classes. Therefore, this work proposes an extension to the Multiresolution Segmentation method (Baatz00), in a way that several morphological attributes can be considered in the region growing process. In order to select a segmentation quality assessment metric to be used in the evaluation of the proposed segmentation algorithm, a study on eight metrics available in the literature was conducted. This study aimed at assessing the relative performance of the quality metrics and to verify which of them presented the higher correlation with the human perception of segmentation quality. Eight shape attributes were then chosen to compose the heterogeneity criterion and the quality of segmentations using one shape attribute at a time was compared with the color only based segmentation. After that, the impact of using pairs of morphological attributes was also evaluated. The experiments were performed over fifteen classes of interest present in twelve different images, representing application areas such as remote sensing, microscopy and medical images. The results confirm the importance of including morphological attributes in the segmentation process and promote an interesting discussion about future works.

\section{Keywords}

Image Processing; Image Segmentation; Segmentation Quality Assessment; Shape Features. 


\section{Sumário}

1 Introdução 16

$\begin{array}{ll}\text { 1.1. Objetivos } & 18\end{array}$

$\begin{array}{ll}\text { 1.2. Estrutura da Dissertação } & 18\end{array}$

2 Fundamentos Teóricos 20

2.1. Técnicas de Segmentação 20

2.1.1. Segmentação por Descontinuidades 21

2.1.2. Limiarização 21

2.1.3. Segmentação por Regiões 23

2.1.4. Segmentação Multiresolução 25

2.2. Características dos Objetos de Imagem 28

2.2.1. Comprimento de borda 29

2.2.2. Perímetro 29

2.2.3. Ajuste de Elipse $\quad 30$

2.3. Qualidade da Segmentação 32

2.4. Métricas de Discrepância 35

2.4.1. Reference Bounded Segments Booster (RBSB) 36

2.4.2. Larger Segments Booster (LSB) 37

2.4.3. Janssen (JAN) 37

2.4.4. Sobreposição Espacial (SE) 38

2.4.5. Diferença de Área Relativa Absoluta (DARA) 38

2.4.6. Distância de Perímetro Absoluta Simétrica Média (DPASM) 38

2.4.7. Distância de Perímetro Simétrica RMS (DPSRMS) 39

2.4.8. Distância de Perímetro Absoluta Simétrica Máxima (DPASMx) 39

2.5. Correlação de Ordem 39

2.6. Técnicas de Otimização 41

2.6.1. Algoritmos Genéticos (AG) 41

2.6.2. Generalized Pattern Search (GPS) 42

3 Segmentador Multiresolução Estendido 46 
3.1. Processo de Segmentação 46

3.2. Atributos de Forma 47

3.3. Implementação 50

3.3.1. Protótipo 50

3.3.2. Ambiente 52

4 Avaliação Experimental 53

4.1. Avaliação das Métricas de Discrepância 53

4.1.1. Banco de Dados 54

4.1.2. Correlação de Ordem entre Métricas de Discrepância 55

4.1.3. Similaridade entre as Soluções do AG para Diferentes Métricas 57

4.1.4. Consistência entre as Avaliações dos Observadores Humanos 59

4.1.5. Métricas Consistentes com a Percepção Humana 61

4.2. Avaliação dos Atributos de Forma na Segmentação 63

4.2.1. Banco de Imagens 64

4.2.2. Otimização dos Parâmetros de Segmentação 68

4.2.3. Segmentação sem Atributos de Forma 70

4.2.4. Segmentação com um Único Atributo de Forma 71

4.2.5. Segmentação com Dois Atributos de Forma 90

5 Conclusões 95

$\begin{array}{ll}\text { Referências Bibliográficas } & 98\end{array}$

$\begin{array}{ll}\text { Apêndice A } & 104\end{array}$ 


\section{Lista de figuras}

Figura 2.1: Limiar $L$ definido sobre o histograma de uma imagem.

22

Figura 2.2: Comprimento de borda de um círculo mostrado em verde (pixels de borda em cinza).

Figura 2.3: Cálculo do perímetro para um círculo e um quadrado de 10x10 (pixels de borda em cinza).

Figura 2.4: Elipse ajustada a um objeto de imagem. 32

Figura 2.5: Esquema de segmentação e avaliação. 33

Figura 2.6: Conceitos utilizados nas métricas de discrepância. $\quad 36$

Figura 2.7: Exemplo do cálculo gráfico da correlação de Kendall. $\quad 40$

Figura 2.8: Estrutura básica dos AG. 42

Figura 2.9: Fluxograma do algoritmo GPS (Mathworks11, adaptado). 43

Figura 2.10: mesh ao redor do ponto xk.

Figura 3.1: Linha de comando para a chamada do programa XMRS. $\quad 51$

Figura 4.1: Imagem Quickbird. 54

Figura 4.2: Grupos de referências (a) homogêneas,

(b) heterogêneas e (c) mescladas.

Figura 4.3: Exemplos das figuras de segmentações ordenadas pelos observadores humanos.

Figura 4.4: Histogramas da correlação de ordem entre 25 observadores para três grupos de resultados de segmentações.

Figura 4.5: Histogramas da correlação de ordem entre as métricas de discrepância definidas na Seção 2.4.

Figura 4.6: Histogramas da correlação entre cada métrica de discrepância e 25 observadores com relação à qualidade da segmentação no grupo homogêneo.

Figura 4.7: Imagens de sensoriamento remoto: (a) árvores,

(b) refinaria e (c) telhados, com respectivas referências.

Figura 4.8: Imagem de microscopia: cristais de hematita (minério de ferro) e referências da classe lamelar (direita) e granular (abaixo). 
Figura 4.9: Imagem de ressonância magnética e respectiva referência. 66 Figura 4.10: Imagem de raios $X$ da mão e referências das classes falange média (acima), falange proximal (centro) e metacarpo (abaixo). 67 Figura 4.11: Imagens do banco de avaliação de segmentações: (a) sapo, (b) estrela-do-mar, (c) suricato, (d) pedras, (e) lagarta e (f) ovos, com respectivas referências. 68

Figura 4.12: Avaliação dos parâmetros de segmentação.

Figura 4.13: Comparação entre as avaliações das segmentações com um único atributo de forma e as com apenas a cor para as referências das imagens de sensoriamento remoto.

Figura 4.14: Comparação entre as avaliações das segmentações com um único atributo de forma e as com apenas a cor para as referências da imagem de microscopia.

Figura 4.15: Comparação entre as avaliações das segmentações com um único atributo de forma e as com apenas a cor para a referência da imagem de TC.

Figura 4.16: Comparação entre as avaliações das segmentações com um único atributo de forma e as com apenas a cor para as referências da imagem de raios $X$.

Figura 4.17: Comparação entre as avaliações das segmentações com um único atributo de forma e as com apenas a cor para as referências das imagens do banco de imagens.

Figura 4.18: Média e desvio-padrão das avaliações para os experimentos da imagem Telhados.

Figura 4.19: Média e desvio-padrão das avaliações para os experimentos da imagem Fígado.

Figura 4.20: Média e desvio-padrão das avaliações para os experimentos da imagem Estrela-do-mar.

Figura 4.21: Média e desvio-padrão das avaliações para os experimentos da imagem Ovos.

Figura 4.22: Média e desvio-padrão das avaliações para os experimentos da imagem Pedras.

Figura 4.23: Porcentagem de ganho entre o mínimo das 
avaliações das segmentações com 1 atributo de forma e a baseada somente na cor.

Figura 4.24: (a) Imagem original e (b) segmentação baseada apenas na cor. As imagens (c), (d) e (e) apresentam as segmentações com os três atributos de forma que obtiveram melhor desempenho: Fator de Forma Circular, Compacidade e Bulkiness.

Figura 4.25: (a) Imagem original e (b) segmentação baseada apenas na cor. As imagens (c), (d) e (e) apresentam as segmentações com os três atributos de forma que obtiveram melhor desempenho: Compacidade, Bulkiness e Fator de Forma Circular.

Figura 4.26: (a) Imagem original e (b) segmentação baseada apenas na cor. As imagens (c), (d) e (e) apresentam as segmentações com os três atributos de forma que obtiveram melhor desempenho: Fator de Forma Circular, Suavidade e Compacidade.

Figura 4.27: (a) Imagem original e (b) segmentação baseada apenas na cor. As imagens (c), (d) e (e) apresentam as segmentações com os três atributos de forma que obtiveram melhor desempenho: Suavidade, Fator de Forma Circular e Excentricidade.

Figura 4.28: (a) Imagem original e (b) segmentação baseada apenas na cor. As imagens (c), (d) e (e) apresentam as segmentações com os três atributos de forma que obtiveram melhor desempenho: Suavidade, Anisometria e Fator de Forma Circular.

Figura 4.29: Comparação entre as avaliações das segmentações obtidas com um e com dois atributos de forma para as referências das imagens de sensoriamento remoto.

Figura 4.30: Comparação entre as avaliações das segmentações obtidas com um e com dois atributos de forma para as referências da imagem de microscopia. 
Figura 4.31: Comparação entre as avaliações das segmentações obtidas com um e com dois atributos de forma para a referência da imagem de TC.

Figura 4.32: Comparação entre as avaliações das segmentações obtidas com um e com dois atributos de forma para as referências da imagem de raios $X$.

Figura 4.33: Comparação entre as avaliações das segmentações obtidas com um e com dois atributos de forma para as referências das imagens do banco de imagens.

Figura 4.34: (a) Imagem original e (b) segmentação com o melhor atributo de forma da etapa anterior. (c) e (d) apresentam as segmentações com os dois atributos de forma de melhor desempenho na combinação com o atributo Suavidade:

Retangularidade e Bulkiness.

Figura A.1: (a) Imagem original e (b) segmentação baseada apenas na cor. As imagens (c), (d) e (e) apresentam as segmentações com os três atributos de forma que obtiveram melhor desempenho: Compacidade, Fator de Forma Circular e Roundness.

Figura A.2: (a) Imagem original e (b) segmentação baseada apenas na cor. As imagens (c), (d) e (e) apresentam as segmentações com os três atributos de forma que obtiveram melhor desempenho: Fator de Forma Circular, Compacidade e Bulkiness.

Figura A.3: (a) Imagem original e (b) segmentação baseada apenas na cor. As imagens (c), (d) e (e) apresentam as segmentações com os três atributos de forma que obtiveram melhor desempenho: Compacidade, Fator de Forma Circular e Bulkiness.

Figura A.4: (a) Imagem original e (b) segmentação baseada apenas na cor. As imagens (c), (d) e (e) apresentam as segmentações com os três atributos de forma que obtiveram melhor desempenho: Fator de Forma Circular, Suavidade e 
Roundness.

Figura A.5: (a) Imagem original e (b) segmentação baseada apenas na cor. As imagens (c), (d) e (e) apresentam as segmentações com os três atributos de forma que obtiveram melhor desempenho: Fator de Forma Circular, Suavidade e Compacidade.

Figura A.6: (a) Imagem original e (b) segmentação baseada apenas na cor. As imagens (c), (d) e (e) apresentam as segmentações com os três atributos de forma que obtiveram melhor desempenho: Compacidade, Isometria, e Fator de Forma Circular.

Figura A.7: (a) Imagem original e (b) segmentação baseada apenas na cor. As imagens (c), (d) e (e) apresentam as segmentações com os três atributos de forma que obtiveram melhor desempenho: Compacidade, Retangularidade e Anisometria. 110 Figura A.8: (a) Imagem original e (b) segmentação baseada apenas na cor. As imagens (c), (d) e (e) apresentam as segmentações com os três atributos de forma que obtiveram melhor desempenho: Fator de Forma Circular, Retangularidade e Bulkiness.

Figura A.9: (a) Imagem original e (b) segmentação baseada apenas na cor. As imagens (c), (d) e (e) apresentam as segmentações com os três atributos de forma que obtiveram melhor desempenho: Fator de Forma Circular, Compacidade e Retangularidade.

Figura A.10: (a) Imagem original e (b) segmentação baseada apenas na cor. As imagens (c), (d) e (e) apresentam as segmentações com os três atributos de forma que obtiveram melhor desempenho: Fator de Estrutura, Anisometria e Roundness. 


\section{Lista de tabelas}

Tabela 2.1: Medidas usadas em avaliações empíricas. 34

Tabela 2.2: Medidas usadas em avaliações empíricas. 35

Tabela 4.1: Coeficiente de correlação de ordem de Kendall (\%)

para o grupo de referências homogêneas. 56

Tabela 4.2: Coeficiente de correlação de ordem de Kendall (\%)

para o grupo de referência mescladas. 56

Tabela 4.3: Coeficiente de correlação de ordem de Kendall (\%)

para o grupo de referências heterogêneas. 56

Tabela 4.4: Valores dos parâmetros obtidos para o grupo homogêneo. 58

Tabela 4.5: Valores dos parâmetros obtidos para o grupo mesclado. 58

Tabela 4.6: Valores dos parâmetros obtidos para o grupo heterogêneo. 59

Tabela 4.7: Parâmetros estimados para uma segmentação

baseada somente na cor. 